\title{
L'HOMME L'Homme
}

Revue française d'anthropologie

157 | janvier-mars 2001

Représentations et temporalités

\section{Clivages et dérapages}

Première et deuxième générations d'Africains en banlieue parisienne

\section{Charles-Henry Pradelles de Latour}

\section{(2) OpenEdition}

\section{Journals}

Édition électronique

URL : http://journals.openedition.org//homme/94

DOI : 10.4000/lhomme.94

ISSN : 1953-8103

Éditeur

Éditions de l'EHESS

\section{Édition imprimée}

Date de publication : 1 janvier 2001

Pagination : 151-166

ISBN : 2-7132-1357-6

ISSN : 0439-4216

Référence électronique

Charles-Henry Pradelles de Latour, "Clivages et dérapages », L'Homme [En ligne], 157 | janvier-mars 2001, mis en ligne le 23 mai 2007, consulté le 20 avril 2019. URL : http://journals.openedition.org/ Ihomme/94 ; DOl : 10.4000/lhomme.94 


\title{
Clivages et dérapages \\ Première et deuxième générations d'Africains \\ en banlieue parisienne
}

\section{Charles-Henry Pradelles de Latour}

Au Centre d'action éducative de la PJJ de Saint-Denis

\begin{abstract}
"La situation migratoire constitue, en elle-même, dans bien des domaines une situation de rupture et il ne s'agit pas de voir partout une reproduction des réalités propres à la société africaine d'origine. " Mahamet Timera.
\end{abstract}

Au début du XX ${ }^{\text {e }}$ siècle, dans un de ses ouvrages ${ }^{1}$ Franz Boas note que les enfants d'immigrés européens arrivés aux États-Unis s'éloignent ostensiblement des coutumes et façons de penser de leurs parents, bien que celles-ci aient été vivement entretenues dans des communautés fermées ne regroupant chacune que des Siciliens, des Calabrais, des Polonais ou des Juifs. Mon activité de médiateur, exercée pour le compte des juges d'enfants du Tribunal de Grande Instance de Nanterre auprès de familles d'immigrés africains ayant fait l'objet d'un signalement grave ou bénin, m'a amené, à la suite de Marcus Lee Hansen (1940) et de Roger Bastide (1970), à corroborer cette hypothèse. Au cours de cette expérience qui, depuis dix ans, me fait côtoyer une quinzaine de familles chaque année, j'ai pu vérifier combien les enfants d'immigrés étaient fortement coupés des traditions et des modes de penser de la société d'origine de leurs parents, et qu'ils étaient plus insérés qu'on ne le pense dans la société d'accueil qui les a vus naître. Afin de rendre compte du décalage qui sépare la première de la deuxième génération d'immigrés africains résidant dans les Hauts-deSeine, nous allons tenter de cerner les clivages spécifiques qui s’opèrent entre les deux sociétés de référence de ces générations, puis nous verrons en quoi l'absence de clivages est facteur de dérapages.

Les immigrés, qui dans l'ordre social représentent le paradigme de l'autre, sont le plus souvent appréhendés sans nuance. Ils sont soit autres, résolument étrangers, par conséquent divisés - chaque groupe constituant de par son origine une singularité irréductible -, soit nos semblables, citoyens ou prochains, et unifiés par la reconnaissance de la dignité attribuée a priori à tout homme. "Ces deux formes élémentaires de la relation à l'autre» (Schnapper 1988: 33) sous-tendent l'approche respectivement des ethnologues culturalistes qui cherchent à accentuer l'altérité, et

1. Cet ouvrage, intitule The Mind of the Primitive Man, est connu en France par le compte-rendu que Durkheim en a fait dans l'Année sociologique XII, 1909-1912. 
des sociologues universalistes qui postulent, au-delà les particularités de chaque groupe, l'unité commune au genre humain. Ces conceptions contrastées, auxquelles répondent les immigrés africains, à savoir autres de par leur appartenance ethnique, semblables de par leur insertion dans une société fondée sur un droit universel, sont trop exclusives. Aussi ne peuvent-elles que rivaliser sur un plan éthique et obstruer, de ce fait, toute analyse. Bien qu'apparemment généreuses, ces deux approches de l'autre recèlent en effet un «envers » qui s'est tristement illustré dans l'histoire. Lorsqu'on envisage l'autre dans une radicale altérité, les groupes ethniques se hiérarchisent inéluctablement, et les plus dépréciés sont tenus à l'écart par les lois de l'apartheid ou exterminés. Pour ce qui est de la relation à l'autre semblable à soi, elle a souvent servi à justifier des impérialismes et des colonialismes dont la violence ne le cède en rien au point de vue précédent.

Afin de dépasser ces approches extrêmes et leurs contradictions, nous nous proposons de recourir ici à deux concepts issus de la théorie des ensembles, qui présentent l'avantage de pouvoir à la fois s'opposer et se recouvrir en glissant l'un sur l'autre.

\section{Appartenance et inclusion}

Dans la mathématique ensembliste, les éléments appartiennent à un ensemble, et les sous-ensembles y sont inclus. Selon Alain Badiou, qui a redéfini ces concepts en termes d'ontologie, les éléments qui appartiennent à un ensemble font l'objet d'une présentation, tandis que les sous-ensembles inclus dans un ensemble supérieur font l'objet d'une re-présentation (1988: 52). En d'autres termes, l'appartenance pure (sans inclusion), définie uniquement par l'existence de ses éléments, témoigne de la singularité irréductible de ceux-ci, tandis que l'inclusion sans appartenance est purement nominale comme peuvent l'être l'unité et la dignité postulées du genre humain. Si, dans leur exclusivité, l'appartenance et l'inclusion reviennent d'une certaine manière aux deux formes élémentaires de la relation à l'autre, dans la réalité quotidienne ces deux notions se recouvrent en permanence. Tout individu appartient à... et est inclus (présent et représenté) dans une cellule familiale minimale et dans un lieu de résidence, lesquels appartiennent à... et sont inclus dans des communautés plus larges, telles que la région, le pays, etc. Selon cette distribution, on appartient toujours davantage - y étant inclus - aux unités de base, famille et travail, existentielles pour tout un chacun, et l'inclusion l'emporte sur l'appartenance, dans les communautés politiques élargies où l'on est plus représenté que présenté. Ainsi, plus les inclusions s'étendent à de grands ensembles, telle la mondialisation des échanges, plus les appartenances locales et ethniques s'affirment pour revendiquer leur droit à la singularité. Donc, quand l'appartenance prime sur l'inclusion, on fait existentiellement corps avec un groupe, et quand l'inclusion est première, on étend à d'autres son identité. La primauté accordée à l'inclusion ou à l'appartenance va nous aider à définir en quoi les modalités de l'immigration et de l'adoption s'opposent. 
Dès le début de son processus, l'immigration se fonde sur les inclusions. Un immigré qui débarque dans un nouveau pays s'appuie en premier lieu sur les représentations. Les Africains racontent qu’au début de leur séjour à Paris, ils ont dû apprendre à compter en francs français, à lire les panneaux signalétiques dans les transports en commun, à écouter attentivement l'articulation des sons étrangers à leurs langues, et à mémoriser, véritable casse-tête pour eux, les masculin ou féminin de la langue française. Puis, il leur a fallu s'adapter à des contraintes horaires, à un rythme de vie accéléré et à des ordres répétés pour s'intégrer à un lieu de travail. Les immigrés africains acceptaient d'autant mieux ces contraintes étrangères que, dans leurs lieux de résidence, ils avaient des parents et des compatriotes avec lesquels ils retrouvaient leur propre façon de vivre. Ainsi les nouvelles inclusions suivies d'appartenances nouvelles se surajoutent-elles aux anciennes formant, comme en géologie, deux couches clivées, sur lesquelles les immigrés vivent en permanence.

Contrairement à l'immigration, l'adoption débute par l'insertion de l'enfant dans une nouvelle famille, les parents adoptifs ayant à charge ensuite de l'inclure affectivement et culturellement dans leur milieu. Si toutefois l'appartenance et l'inclusion tendent ainsi à se recouvrir, elles n'en effacent pas toujours le passé, car, même dans l'adoption plénière, les enfants finissent par savoir qu'ils ont une autre famille dans laquelle ils sont inclus, faute d'y appartenir. Donc chez les immigrés africains de la première génération ou chez leurs enfants "adoptés» en quelque sorte par la société d'accueil dans laquelle ils sont nés, il existe, entre les modes de vie ancien et nouveau, différents clivages dont nous allons, en les resituant dans leur histoire et dans leur contexte social, cerner la nature.

\section{Clivages des immigrés de la première génération}

L'immigration des Africains en France se distingue en ceci que, dès l'origine, elle a été considérée comme temporaire. "À la différence d'autres flux migratoires, le gouvernement n'a jamais rien fait pour les encourager à venir» (Winthol de Wenden 1988: 181). "Il les a traités comme s'ils ne faisaient que du tourisme" (Sayad 1977: 60). Les premiers arrivés étaient tous des célibataires qui, après avoir un temps vendu leur force de travail au pays d'accueil, rentraient dans leur pays d'origine. Dans les années 60 et 70, les immigrés africains furent employés comme main-d'œuvre non qualifiée par les grandes sociétés industrielles (automobiles et fonderies), les PME et les municipalités. À la suite des vagues de licenciements pour raison économique dans les années 80, ils retrouvèrent du travail dans des entreprises de nettoyage, la restauration collective, les agences de surveillance et les sociétés d'intérim. Les immigrés africains, qui sont passés ainsi d'emplois jugés virils à des tâches plus diversifiées et moins valorisées, n’ont jamais constitué un corps de travailleurs organisés et inscrits dans un syndicat défendant leurs revendications et leurs droits, comme l'avaient fait auparavant les immigrés italiens, espagnols et portugais. On ne trouve pas non plus de projet professionnel associé à leur trajet migratoire (Timera 1996 : 
219). Les Maliens, Mauritaniens et Sénégalais venus en France recherchaient un emploi, non une profession. Quant aux Camerounais, Congolais et ressortis154 sants de la République démocratique du Congo, qui arrivaient en France afin de poursuivre des études après un parcours scolaire plus ou moins long, ils étaient souvent obligés de renoncer à une formation et devaient, pour vivre, accepter des emplois sans qualifications. Dans leur grande majorité, les immigrés africains constituent pour le bénéfice de leurs employeurs « une main-d'œuvre anonyme sans substance sociale» (ibid. : 38) qui remplit son contrat : travail contre salaire minimal. La recherche de la meilleure offre pour une moindre charge a joué dans certains cas en faveur des travailleurs, mais la généralisation des emplois à temps partiel et à horaires décalés - tôt le matin tôt, tard le soir - a avantagé de toutes les manières les patrons des entreprises de nettoyage.

Si les Africains se sont soumis aux exigences des travaux qu'on leur proposait, ils ont su faire valoir leurs droits et leur dignité en réclamant à plusieurs reprises des conditions de logement meilleures. Les immigrés de la première génération arrivaient en France en suivant une filière parentale, l'aîné installé envoyant le billet d'avion à un frère cadet pour que celui-ci puisse venir le remplacer plus tard. Regroupés ainsi par familles et par villages, ils furent, dans un premier temps, victimes des marchands de sommeil qui leur louaient des chambres insalubres qu'ils occupaient à tour de rôle. Leur première revendication sera de demander aux pouvoirs publics la construction de foyers où ils pourront avoir des chambres convenables. Dans les années 70, ils formeront des comités qui, pour obtenir des conditions de vie respectables au sein des foyers, feront la grève des loyers pendant cinq ans. Cette étape importante permettra aux immigrés de faire connaître leur volonté d'insertion et de légitimer leur mouvement auquel de nombreuses associations et des intellectuels apporteront leur soutien. Le public apprendra alors que les Africains sont très organisés, qu'ils créent des « caisses » de solidarité qu’ils alimentent avec leur salaire. Payé au SMIG, un travailleur immigré parvenait à vivre avec un tiers de son revenu, réservant les deux autres tiers pour contribuer aux caisses de solidarité et aider sa famille restée au pays (Quiminal 1991: 119).

Le trajet migratoire des Africains résidant en banlieue parisienne subit un profond changement au début des années 80 lorsqu'une grande partie d'entre eux profitant des décrets autorisant les ressortissants de tous les pays à mener une vie familiale normale, firent venir auprès d'eux femmes et enfants restés jusque-là au pays. Ces regroupements familiaux, qui s'opéraient dans les quartiers et les cités, encouragèrent une insertion, qui devint à la fois provisoire et durable. Ce début d'intégration se fit au détriment des villages d'origine qui perdaient du même coup une main-d'œuvre féminine mais pas l'argent envoyé régulièrement par les hommes comme s'ils étaient restés célibataires. En revanche, ce déplacement favorisa une vie résidentielle dans laquelle les femmes arrivées en nombre ont joué un rôle déterminant (Quiminal 1995 : 113). Les hommes continuèrent à assister dans les foyers à leurs réunions familiales, et ils mirent sur pied dans leur quartier de nouvelles associations, certaines regroupant les individus d'une même région, 
d'autres, plus larges, accueillant des individus appartenant à plusieurs ethnies, voire parfois même à différents pays africains. Les premières avaient pour objectif de promouvoir le développement d'un village en construisant une école, un dispensaire ou de nouvelles routes ; les secondes envisageaient surtout de recueillir des cotisations pour financer, le cas échéant, le retour des morts au pays. De leur côté, les femmes, libérées des tâches agricoles qu'elles assumaient au village, prirent l'habitude de se retrouver dans les quartiers, les anciennes accueillant les nouvelles et cherchant toutes à s'entraider. Soucieuses de l'éducation des enfants, elles acceptèrent facilement l'aide offerte par les institutions sociales et plus particulièrement par les $\mathrm{AS}^{2}$ qui leur apprirent, entre autres, au grand dam des maris, que les prestations familiales leur revenaient de droit. Les femmes élargirent ainsi leur réseau de communication, devinrent plus autonomes, se mirent au travail à temps partiel, certaines divorcèrent; en somme, elles s'ouvrirent plus rapidement que les hommes aux idéaux de la société d'accueil. Dans les années 90, elles commencèrent à se regrouper en associations plus diverses qui pouvaient comprendre des ménagères et des avocates appartenant aussi bien à l'Afrique qu'au Maghreb. Ces associations défendaient l'égalité des sexes, la reconnaissance de leurs droits et luttaient contre les coutumes qui les maintenaient dans la dépendance de leur lignage (ibid. : 119). Les hommes et les femmes immigrés suivirent ainsi des parcours différents, les premiers préférant l'aide à leur pays d'origine où ils désiraient finir leurs jours, les secondes étant davantage soucieuses de s'intégrer avec leurs enfants dans la société d'accueil. Cette divergence qui ne va pas sans conflit au sein des familles, fait apparaitre que les hommes n'arrivent pas à appartenir à leur terre d'immigration. Mais pourquoi ce clivage est-il si marqué pour les hommes?

Comme le souligne Abdelmalek Sayad (1977: 59) : «Toute étude de l'immigration qui négligerait les conditions d'origine de l'émigré se condamnerait à ne donner du phénomène migratoire qu'une vue partielle et ethnocentrique [...], comme si [l'] existence [de l'émigré] commençait seulement au moment où il arrive en France». Le principal ressort de l'émigration africaine est d'abord d'ordre économique. À négliger cet aspect, on se condamne à un certain aveuglement. Les émigrés africains viennent soit de villages où les ressources sont assurées par une agriculture qui, bien qu'extensive, n'a qu'un faible rendement car elle est toujours pratiquée avec des moyens traditionnels, soit de villes où la masse des non salariés vit au jour le jour de petits commerces et de manutentions occasionnelles. Les travailleurs africains, ruraux ou urbains, qui arrivent à subvenir aux besoins de leur famille, disposent rarement de ressources suffisantes pour payer des impôts et des soins médicaux. Par conséquent, même un SMIG, qu’un autochtone jugerait gagné dans des conditions d'exploitation insupportables, semble une "aubaine ${ }^{3}$ pour un Africain, car son salaire mensuel équivaut à dix fois ce qu'il aurait obtenu en une année dans son pays. Bénéficier d'un salaire régulier, quel qu'en soit le prix, est le principal motif de l'émigration africaine.

2. Acronyme usuel en banlieue pour désigner l'assistante sociale.

3. L’aubain désignait originellement l'étranger, et le droit d'aubaine était leur héritage qui, après leur mort, revenait officiellement au roi. 
Le décalage considérable existant entre l'économie européenne et l'économie africaine explique largement l'écart des rapports sociaux propres à chaque continent. Si, en Europe, le développement est marqué par la découverte de nouvelles technologies et le renouvellement de la hiérarchie sociale des compétences, il est en Afrique essentiellement tributaire des statuts hiérarchiques des lignages (anciens guerriers et captifs - notables et villageois) et de la croissance démographique. Plus une famille est d'un rang élevé et plus elle a d'enfants, plus elle est riche. Si, en Europe, les rapports sociaux dominants se fondent sur la mầtrise politico-économique des technologies de production et de communication, dans les pays africains la reproduction démographique et le renouvellement de la force de travail sont assurés par des systèmes de parenté fortement implantés, dont la méconnaissance conforte la vision ethnocentrique que les Européens ont des immigrés.

Dans les villages africains et même dans les villes, l'ordre social est traditionnellement structuré par les relations de filiation et d'alliance matrimoniale. On sait donc que la filiation dépasse toujours la cellule familiale, père-mère-enfant, en formant des groupes patri- ou matrilinéaires, appelés lignages ou clans, dont l'ancêtre fondateur masculin, féminin ou éponyme peut remonter à plusieurs générations. Par des devoirs envers ses aînés et des droits envers ses cadets, qui resserrent leurs liens de solidarité, tout individu est lié conventionnellement aux membres d'une descendance unilinéaire commune. Ces groupes de filiation sont souvent dirigés par un chef ou un doyen de lignage qui, dans les villages, préside à la répartition des terres cultivables, au partage des récoltes et aux grandes cérémonies ponctuant naissances, mariages et morts. Ce sont eux qui reçoivent les sommes d'argent envoyées par leurs frères ou leurs fils émigrés et les dépensent en fonction des besoins du groupe. Les relations matrimoniales entre les groupes de filiation, qu'il ne faut pas confondre avec les relations conjugales unissant deux époux, sont, pour leur part, concrétisées par le paiement du prix de la fiancée versé par la famille du fiancé à la famille de la future conjointe. Contrairement à ce que les Occidentaux ont souvent pensé, ces transactions matrimoniales n'ont rien à voir avec une transaction commerciale. L'établissement du prix de la fiancée n'est fonction ni de la valeur de la femme échangée, qu'elle soit travailleuse ou non, ni des lois de l'offre et de la demande. Le prix de la fiancée est soit fixe, établi une fois pour toute selon une convention, soit fluctuant et, dans ce cas, il dépend de la richesse du lignage du fiancé. Même si ce prix est devenu vénal dans certaines régions, et plus particulièrement auprès des immigrés détenant un salaire, lorsqu'un homme l'a versé à sa belle-famille, il devient le débiteur de celle-ci. Une femme n'équivaut donc pas à un crédit mais à une dette dont le montant ne peut être recouvré. Un mari est alors subordonné à sa belle-famille, qui est en droit de le sermonner s'il maltraite leur fille. Toutes les relations parentales et sociales sont ainsi empreintes d'une redevance : dette de réciprocité à l'égard des membres de la classe d'âge, dette différée des cadets envers leurs aînés en attendant de les remplacer, dette symbolique entre les alliés et les étrangers. Les Occidentaux manipulent constamment des objets et recherchent le crédit afin de prouver leur autonomie, les Africains, eux, sont quo- 
tidiennement liés aux autres par tout un jeu de redevances et de gratitudes, qui est au fondement de leurs lois et de leurs liens sociaux. D'où les relations d'entraide qu'ils entretiennent même hors de leur communauté et l'appartenance indéfectible à leur village d'origine.

Enfin, rappelons que les Africains qui, pour la plupart d'entre eux, sont originaires de sociétés dites sans écriture, hormis celles qui leur on été apportées, sont beaucoup plus sensibles que les Européens aux logiques et effets de la parole. La communication se trouve fortement investie par la crainte de mal dire (ce qui explique la pratique des longues salutations) et par le souci de se conformer à la volonté du destinataire du message dont on attend en permanence reconnaissance et conseils. On n'utilise pas les mêmes tournures pour s'adresser à un aîné, à un cadet, à un consanguin ou à un allié, à un voisin où à un étranger. De la même manière, un Africain peut très facilement parler en termes de crédit et d'autonomie avec des Européens et ensuite s'exprimer en termes de dettes et d'obligations avec les siens. Cela se peut d'autant mieux que les deux ordres sociaux ne répondent pas aux mêmes modalités. Dans son pays d'accueil, un Africain est surtout "inclus " grâce à un jeu de représentations et par des contrats de travail sans substance sociale (sans projet de formation et de carrière) dont il connaît et respecte les règles; dans le pays d'origine auquel il appartient davantage tout en y étant inclus, il est physiquement et idéologiquement relié aux autres par les lois de la parenté et de l'oralité. C'est pourquoi les immigrés de la première génération bâtissent, quand ils le peuvent, une maison au pays, afin d'y vivre après la retraite le restant de leurs jours, et payent des cotisations pour que leur corps puisse y être enterré par les leurs. Alors que l'inclusion implique un lieu où on est représenté de différentes manières, ce pour un temps donné, l'appartenance implique un ancrage existentiel qui participe de l'éternité. Pour parachever ce survol, précisons que l'Afrique des immigrés à laquelle nous nous référons est plus pauvre et plus traditionnelle que celle des nouvelles classes de salariés vivant dans les capitales africaines, que nous n'avons pas prises en compte car on ne les retrouve pas parmi les immigrés résidant dans les cités des Hauts-de-Seine.

\section{Clivage des immigrés de la deuxième génération}

Le drame des immigrés africains vivant en banlieue parisienne avec leur famille tient au fait qu'ils ne peuvent pas transmettre à leurs enfants leur mode de vie et leur façon de penser. La culture ethnique n'est pas transmissible à la génération suivante, parce que non seulement les initiations et les rites qui la sous-tendent ne peuvent être célébrés en dehors de leur contexte local, mais aussi et surtout parce que la culture africaine est fortement tributaire d'un système de parenté dont l'extension n'est guère transposable d'un pays à l'autre. Dans les villages africains, qui regroupent les lignages des quatre grands-parents et ceux de nombreux alliés, les enfants sont élevés d'un côté par leurs camarades de la même classe d'âge avec lesquels ils partagent jeux, premières activités 
agraires et plaisanteries, et de l'autre par leurs aînés qui leur enseignent ce qui est permis et interdit et par leurs grands-parents qui les abreuvent de contes et leur apprennent à s'exprimer en images à l'aide de proverbes. Avec les grandsmères, les jeunes filles découvrent avant l'âge ce que signifient les règles et comment elles accoucheront. Avec les grands-pères, les garçons sont initiés aux paradoxes de la parole qui, "en léchant la main des deux côtés ", tourne la vérité en mensonge et inversement. Les parents, pour leur part, interviennent essentiellement dans l'ordre moral en récompensant les réussites et en sanctionnant les délits et les échecs par des punitions corporelles si nécessaire, et, dans l'ordre traditionnel, en célébrant les rites et les fêtes requises par le cycle de la vie et celui des saisons. En somme, si en Afrique il faut trois générations au moins pour assurer la transmission d'une tradition dans toute sa complexité, qu'en reste-t-il dans les cités de la banlieue parisienne où les immigrés vivent à la façon occidentale dans la stricte cellule familiale ? Pas grand-chose, outre la relation parents-enfants dont la prégnance redouble - parfois même de façon dangereuse - en l'absence des autres relations de médiation assumées au pays par la classe d'âge et les grands-parents. "Les enfants ne sont plus, ici, les enfants du lignage mais ceux des parents " (Timera 1996 : 108). Cette acculturation implique que, bien souvent, les enfants d'immigrés ne connaissent pas le nom de leur ethnie d'origine. Dans les familles africaines des Hauts-de-Seine où j'ai travaillé, j'ai souvent entendu des enfants dire : "Je ne savais pas que mon père était Soninké ou ma mère Bambara. » Ce sont mes questions qui ont éveillé un intérêt pour la société d'origine et la vie traditionnelle pratiquée au village.

La culture africaine se perd non seulement, comme on vient de le voir, par absence de transmission, mais aussi parce qu'elle est remplacée par la pratique quotidienne d'une langue étrangère, et par les valeurs économiques et idéologiques du pays d'accueil qui sont largement et fortement diffusées par le biais de la télévision ou de l'enseignement scolaire. La télévision, qui est allumée du matin au soir dans la pièce centrale de nombre de familles de la banlieue, inculque par ses émissions un langage et une pensée généralisée, convenue, et alimentent l'univers fantasmatique des jeunes générations. Ainsi, des enfants d'origine africaine, nés en France, m’ont raconté qu'ils avaient rêvé, l'un "de princesses et de châteaux ", l'autre de "dinosaures galopant à fond la caisse ", et le troisième de "sorcières portant un Tshirt noir fluorescent ». Quant à la formation scolaire, chacun sait qu'elle constitue pour la pensée et les comportements une puissance d'uniformisation d'autant plus forte qu'elle est vivement valorisée par les parents constituant la première génération d'immigrés, et qu'elle est soutenue par son accessibilité à tous. La majorité des familles d'immigrés expliquent qu'elles ont fait venir leurs enfants en France pour bénéficier de la gratuité de l'école. Ceux-ci sont donc progressivement déconnectés du système de pensée de leur parents, fondé sur les dettes et le jeu des solidarités, par l'apprentissage de la langue et de l'écriture qui détermine un mode de classement et des règles de raisonnement spécifiques. Ils en sont encore davantage éloignés par un système de notes favorisant les meilleurs, par une idéologie de compétition largement entretenue par les jeux électroniques qui fonctionnent selon 
des règles binaires - plus/moins, dedans/dehors, bon/méchant, vie/mort -, et par les sports collectifs tels le foot et le basket dont la pratique professionnelle constitue un idéal d'insertion achevé.

Si les parents ont bénéficié d'un salaire minimum en dépit de conditions de travail difficiles, leurs enfants pensent de façon occidentale et perçoivent, eux, leur marginalité économique. Certains enfants que j'ai côtoyés se sont plaints ouvertement de ne pas avoir de console ou de VTT comme leurs camarades de classe, et certains adolescents de ne pas avoir assez d'argent pour acheter des «sapes». D’autres, sans le dire, méprisent le métier de leurs parents lorsqu'ils affirment: "Je ne serai jamais éboueur ou balayeur." Du même coup, ces enfants portent fréquemment un regard condescendant sur les conditions de vie dans leur pays d'origine. "Là-bas, ce n'est pas génial ». - Ah oui ! Qu'est-ce qui n'est pas génial ?" Les réponses varient, mais on retrouve toujours les mêmes thèmes: la chaleur, les maladies, les moustiques, la misère. D'aucuns disent même : "C'est crad, en saison des pluies tu as de la boue partout». Ainsi le retour, envié par les immigrés de la première génération, devient-il une menace et une punition pour ceux de la seconde. "Si tu ne veux pas aller à l'école, tu vas aller vivre au pays. Tu verras ce que c'est la vie. »Cette sanction est d'autant plus redoutée que les enfants savent qu'elle a déjà été appliquée à plusieurs de leurs aînés, pour le meilleur dans certain cas et pour le pire dans d'autres.

Sur le plan scolaire, les enfants d'immigrés que j'ai rencontrés suivent avec quelques difficultés le cursus qui leur est proposé car ils souffrent d'un handicap au niveau de la langue et du savoir à acquérir. Ils ont notamment du mal à apprendre l'histoire qui ne fait pas partie de la mémoire de leurs parents : «C'est quoi la Grande Guerre?» - «Pourquoi on nous apprend les momies des Égyptiens ?» - «Ah! La Révolution française, c'est le 14 juillet; je ne savais pas. » Les études sont fréquemment subies passivement sans projet professionnel à la clé: "Je n'ai jamais pensé à ce que je ferai plus tard. " J'ai maintes fois remarqué, à la suite de Catherine Quiminal, que les filles, qui peinent souvent plus que leurs frères au départ, finissent mieux à l'arrivée : "Paradoxalement, il y a une urgence (consensuelle) d'autonomie pour les garçons à laquelle les filles ne sont pas soumises » (Quiminal 1997 : 9). L'indépendance est donnée aux fils mais pas aux filles. Comme l'émancipation de celles-ci est, pour leurs parents, subordonnée à leur projet matrimonial, elles le retardent en étudiant. Les filles arrivent ainsi à l'université avant les garçons. Si l'insertion professionnelle des uns et des autres n'est pas toujours bien définie, ils manifestent tous le désir de vivre en France, à l'exception de quelques-uns qui ont vécu leurs premières années en Afrique dans un milieu familiale serein. La réponse habituelle est «Au pays, j’irai faire des séjours, mais je n'y resterai pas.» Ce langage, ainsi que leur façon d'être attestent qu'ils appartiennent de facto à leur pays d'accueil.

Mais alors, quel lien les immigrés de la seconde génération ont-ils avec la culture de leur pays d'origine ? En dehors de la langue vernaculaire toujours moins bien parlée par les benjamins, les enfants d'immigrés africains gardent tout 
d'abord quelques habitus liés à la parenté : l'obéissance au père dont l'autorité est en principe incontestable - mais pas incontesté dans la pratique -, le respect des aînés et la solidarité des membres d'une fratrie, davantage assurée par les garçons que par les filles. En outre, garçons et filles pensent se marier et de fait se marient avec un partenaire proche d'eux. Enfants et parents partagent aussi une cuisine et une commensalité qui tendent peu à peu à changer. Pâtes et frites reviennent fréquemment dans la composition du plat principal, et dans certaines familles on ne mange plus à même le plat commun. Rappelons que de leur côté les filles apprennent aussi le tressage des cheveux crépus et le tissage des cheveux lisses au cours de séances de travail à domicile qui durent plusieurs heures. Ces habitus, typiquement africains, ne rapprochent pas pour autant les enfants de leurs parents restés au pays. À la suite des séjours effectués au village, les enfants d'immigrés livrent leurs impressions de manière contradictoire. Au début ils disent : "Oui, c'était bien là-bas. Tout le monde me connaît (fils d'un tel et d'une telle)", et ils évoquent avec quelques mots chaleureux leur grandmère qu'ils ne connaissaient pas: "Elle m'aime beaucoup, elle m'a toujours donné raison. " Puis, si on insiste, d'autres impressions révèlent quelques déceptions. "Mes frères voulaient tous mes habits." - «Mes cousins m'ont fait la jalousie. » - «Les travaux des champs avec la houe, c'est difficile. Toute la journée on ne fait rien, ça manque d'activité. » Un jeune Bamiléké m’a confié : «Je suis dans ma famille africaine comme un étranger. Le rite qu'ils ont fait pour la naissance d'un petit cousin, ça ressemble à du n'importe quoi. »

Les immigrés de la seconde génération appartiennent donc à leur pays d'accueil et sont seulement inclus par le biais de représentations et de quelques attitudes qu'ils partagent avec leur pays d'origine. Mais cette appartenance n'est toutefois pas complète car, du fait de la marginalité économique de leurs parents, les enfants d'immigrés ne bénéficient pas des mêmes conditions de vie et des mêmes possibilités de formation que les enfants d'autochtone. C'est la raison pour laquelle les adolescents se présentent fréquemment comme citoyens d'un pays étranger : "Je suis Malien, Sénégalais ou Camerounais ». La citoyenneté a, pour eux, une valeur d'altérité que n’a pas l'ethnie. Par conséquent, d'une génération à l'autre, le rapport des immigrés au pays d'origine est inversé. Tandis que ceux de la première génération regardent toujours vers l'Afrique et le retour au pays parce qu'ils ne sont liés à la société d'accueil que par le biais de conventions acceptées, ceux de la deuxième génération appartiennent résolument au pays où ils ont été élevés et ne sont inclus dans celui de leur parent que par une histoire racontée, déconnectée de leur propre vécu. Pour assumer ces clivages inversés, il n'est pas rare de voir parents et enfants s'adonner à une pratique religieuse dont le système de valeurs unifié les rapproche tout en leur conférant, de façon légitime, l'altérité dont ils ont besoin pour se démarquer des autochtones. Ainsi, pour de nombreuses familles d'immigrés africaines, les religions musulmane ou chrétienne (catholique, évangélique, chrétien céleste ou kibangiste) sont à la fois le sceau d'une ethnicité manquante et le rachat, par un universalisme affiché, d'une dignité souvent bafouée dans la quotidienneté. 


\section{Les dérapages de la première génération d'immigrés}

$\mathrm{Si}$, comme nous le soutenons, la norme des immigrés africains de la première génération tient au maintien du clivage entre l'appartenance fondamentale à la société d'origine et l'inclusion dans le pays d'accueil, les inadaptations ont pour cause l'absence de clivage entre ces deux modalités sociales. Ainsi, en tant que médiateur, j'ai rencontré des hommes et des femmes éprouvés soit parce que, trop arrimés à leur héritage social, ils n'arrivent pas à s'insérer dans la société d'accueil, soit au contraire parce que, trop désireux d'être entièrement intégrés dans cette dernière, ils nient, dangereusement pour eux et leurs enfants, leur appartenance ethnique d'origine. Ces dérapages définis ici à partir d'une distinction purement sociologique, et non à partir d'une clinique, comprennent toutes deux, dans leurs manifestations aiguës, des sentiments de persécution. L'auto-accusation et la mélancolie découlant d'une extrême culpabilité, étaient absentes, du moins jusqu'à une date récente, de l'observation faite dans les hôpitaux africains (Collomb \& Zwingelstein 1963). Tandis que les premières manifestations de persécution typiquement traditionnelles s'expriment par les croyances en la sorcellerie, par les maraboutages ou par la soumission à une entité surnaturelle, génies (djinn) ou ancêtres, voire par une malédiction paternelle transmise à travers les générations, les manifestations de persécution étroitement associées à une folie des grandeurs se rapprochent sensiblement de la description de la paranoïa connue de la psychiatrie européenne.

La première forme d'inadaptation, qui s'inscrit dans le champ de l'ethnopsychiatrie fondée par Georges Devereux, appelle, selon cet auteur, une intervention particulière définie par la culture ethnique à laquelle appartient le patient.

Ainsi, dans La psychothérapie d'un Indien des Plaines, Devereux (1982:262) affirme avoir représenté pour son patient une déité protectrice que les Indiens, enclins à l'apitoiement sur soi, invoquent traditionnellement pour reprendre confiance en eux. Donc, abstraction faite de ce que ce patient est venu en France avec l'armée américaine lors de la Guerre de 1914-1918 et qu'il appartient à une société indienne sérieusement acculturée depuis la fin du XIX ${ }^{e}$ siècle, le thérapeute prétend que c'est parce qu'il a su jouer sur un levier ethnique essentiel que cette psychothérapie a réussi. «Le fait que nous nous soyons immédiatement adaptés au double rôle d'esprit gardien donneur de rêves et de spécialiste indigène de l'interprétation des rêves, a contribué à rendre des pulsions, qui étaient latentes, plus soumises au contrôle et à la direction du moi " (ibid. : 180) "... et à faire accepter un idéal du moi conforme au modèle de la tradition" (sic) (ibid.: 116). Dans le compte rendu des séances quotidiennes, que Georges Devereux a le mérite de donner in extenso, il apparaît que l'Indien a effectivement accepté cette interprétation proposée par son thérapeute. Toutefois, en y regardant de plus près, on s'aperçoit que le patient est capté non pas par la connaissance que ce dernier a de sa culture, mais par ce à quoi il croit. D'où la question posée, un jour, à brûle-pourpoint : "À quelle Église appartenez-vous?» Surpris par cette incursion hors de l'ordre traditionnel, l'ethnopsychiatre répond : "Je n'appartiens à aucune Église, je crois simplement à l'action bonne" (ibid. : 226). Aussi, lorsque, trois semaines plus tard, des signes d'amélioration induits par l'iden- 
tification du patient à son thérapeute apparaissent, l'Indien, qui appartient à une secte protestante particulièrement rigide, est heureux d'annoncer à son bienfaiteur : «Maintenant, je n'ai plus besoin d'aller à l'office (ibid. : 332). Puis, quelque temps après, il réitère cette assertion à la suite d'un petit contretemps marqué par une hésitation : «En une nuit, vous m’avez détourné de toutes les religions. Personnellement je crois que c'est mal. C'est une bonne chose. Mon esprit est maintenant détourné de la religion pour le restant de mes jours" (ibid. : 393). Le thérapeute, qui est - on s'en doute - désarçonné par la vigueur de ces propos, suggère à son patient qu'il a démystifié non pas tant la religion chrétienne que la déité protectrice que lui, psychiatre, a représentée pour lui. Une psychothérapie freudienne n’implique-t-elle pas un processus de démystification qui constitue l'effet libératoire de la cure? Certes, mais il convient de noter que, pour ce faire, le patient s'est appuyé sur un trait culturel qui n'appartenait pas à sa tradition, mais à celle du thérapeute.

Cet exemple, pris à dessein dans la pratique même du fondateur de l'ethnopsychiatrie, atteste qu'une thérapie est, toujours transculturelle car le transfert est généralement orienté du patient vers le thérapeute, et non l'inverse, sauf, bien sûr, en cas de psychose (Allouch 1994: 505 sq.) - mais c'est là un tout autre problème. Il est intéressant de voir que, dans cette psychothérapie rapportée par Devereux (1982 : 390-398), l'Indien est arrivé après un mois de traitement intensif à faire une interprétation d'un de ses rêves tout à fait remarquable en termes de transfert freudien. Après avoir raconté qu'il avait rêvé de deux femmes dont l'une, bonne et généreuse, cherchait à le rapprocher d'une autre qui lui voulait du mal, l'Indien a fort justement repéré que la première représentait son thérapeute, ami des Indiens, et que la subordination à la seconde femme symbolisait la dépendance de ce thérapeute au directeur de l'hôpital psychiatrique, véritable détenteur du pouvoir, et qui, seul, pouvait autoriser le patient à sortir définitivement de son enfermement. La preuve est ainsi donnée, par le biais de cette psychothérapie, que l'Indien a fini par interpréter ses rêves à la manière occidentale.

Il n'est donc pas nécessaire d'être un agent de la culture d'origine du patient pour que la cure soit efficace, il suffit pour cela que chacun des partenaires reste à sa place. C'est à cette condition que le clivage des cultures officie en tant que levier dans une thérapie.

L'expérience que Marie-Cécile Ortigues a menée au centre hospitalier de Fann à Dakar auprès de jeunes Africains en difficulté scolaire a montré qu'une pratique occidentale de la psychothérapie n'excluait pas celle des marabouts (1976: 145339). Pour que la séparation entre ces deux modalités sociales s'effectue, les deux thérapies peuvent coexister mais ne doivent pas être confondues. Le discours démystificateur d'un psychothérapeute ou d'un médiateur ${ }^{4}$, formulé dans la langue du pays d'accueil, est beaucoup plus clair s'il ne se confond pas avec celui des tradipraticiens et s'il peut justement contribuer à séparer ces deux modalités sociales afin que les immigrés se réinsèrent dans leur clivage.

4. Nous préciserons dans un prochain article en quoi l'activité du médiateur est différente de celle du psychothérapeute. Le médiateur qui se déplace dans les familles et travaille en étroite relation avec les éducateurs, est en premier lieu un pacificateur. Étant donné que cette activité implique un suivi marqué par des entretiens réguliers, il arrive parfois qu’à la faveur d’un transfert fait sur lui le médiateur soit occasionnellement pris pour un psychothérapeute, mais ce rôle ne lui est donné que par surcroît, il n'est pas intrinsèque à sa fonction. 
Les immigrés africains qui prétendent vouloir s'intégrer totalement à la sociétés d'accueil sont beaucoup plus troublants que ceux qui se disent ensorcelés, car ils se comportent souvent comme s'ils étaient au-dessus des lois, d'où leurs critiques parfois virulentes des juges et des travailleurs sociaux, et leurs plaintes incessantes d'être victimes de l'incompréhension. Ces manifestations de la persécution sont particulièrement difficiles à traiter, d'une part, parce qu'on arrive mal à différencier dans leurs propos le réel de l'imaginaire et, d'autre part, parce qu'on est dans l'impossibilité de passer d'une culture à l'autre. Ainsi, lorsque j'ai essayé de leur parler de leurs origines, ces hommes m'ont souvent répondu de façon très contradictoire: "Vous ne pouvez rien comprendre à l'Afrique parce que vous êtes Blanc»; et : "Si vous connaissez bien l'Afrique, vous avez dû voir qu'il y a là-bas des voitures et des télévisions comme ici». Donc, tout ou rien. Ces hommes se présentent presque toujours comme des pères parfaits, qui ont tout donné à leurs fils et à leurs filles pour qu'ils puissent venir en France faire des études et avoir un projet professionnel digne de ce nom - avocat, médecin ou député, voire président de la République. Aussi ne comprennent-ils pas que, saturés d'autorité et de demandes impossibles, ceux-ci les fuient ou les contestent en commettant des délits. Si leurs enfants vont mal, c'est qu'ils ont été influencés par les autres enfants du quartier ou qu'ils ont été incompris à cause de leur couleur de peau. C'est là qu'on voit à quel point l'absence de clivage entre les deux modes de vie, africain et européen, peut faire mal, et à quel point leur confusion est la principale source du racisme. Ces cas de persécution, assez fréquents, sont souvent plus difficiles que ceux qui s'expriment sous couvert des croyances traditionnelles, car on n'a pas la possibilité de ré-instaurer le clivage, sinon de façon très exceptionnelle.

Les cas d'inadaptations dont nous venons de faire état ont été choisis en raison de leur rejet extrême de la distinction existant entre les deux modalités de vie, appartenance au pays d'origine et inclusion dans la société d'accueil, qui constitue la norme pour les immigrés de la première génération 5 .

\section{Dérapages des enfants d'immigrés}

Les maladies, qui traditionnellement frappent les enfants en Afrique, se retrouvent parfois en terre d'immigration. Ainsi, j'ai rencontré dans une famille luba venant du Kasai (République démocratique du Congo) une fillette accusée de sorcellerie par la femme de son frère aîné, et, dans une famille bambara des bords du fleuve Sénégal, un enfant walidjou, "un envoyé d'Allah ». L'accusation de sorcellerie m'a d'autant plus étonné qu'en Afrique occidentale les personnes accusées de sorcellerie sont toujours des adultes, mais cette accusation portant sur des enfants s'est désormais répandue à Kinshasa et dans d'autres villes où elle fait des ravages. Imagine-t-on la déréliction et le drame que peut ressentir un enfant accusé d'intentions purement maléfiques. En revanche, les enfants affec-

5. Il existe naturellement bien d'autres pathologies dont nous n'avons pas cherché à faire l'inventaire, car ce sont leurs aspects proprement liés à l'immigration qui nous intéressent ici. 
tés d'une somnolence mentale sont souvent considérés comme des représentants de Dieu ou des figures d'ancêtres à l'instar des enfants nit ku bon (Zempléni \& 164 Rabain 1965). Il existe aussi des enfants très perturbés par la persécution de leurs parents et qui parlent de leur souffrance en termes de sorcellerie ou de génies, mais de façon mal assurée, avec des mots qui n’ont plus la même valeur que celle que leur accorde la première génération d'immigrés. Ainsi le sorcier ou la sorcière ne sont plus liés, pour eux, à une société d'anthropophages mais à un au-delà indéterminé, une sorte d'abîme insondable. Hormis ces quelques cas spécifiques, les enfants d'immigrés présentent des pathologies universellement connues de la pédiatrie, qui sont traitées d'abord dans les dispensaires et les centres médicaux psychologiques et pédagogiques, puis parallèlement ou plus tard par les marabouts et les guérisseurs de la région parisienne.

Les enfants qui, pour diverses raisons, ont passé en Afrique leurs premières années avant de rejoindre leurs parents en France, à la suite d'un regroupement familial, sont en fait à cheval sur les deux générations. Leur double appartenance aux sociétés et d'origine et d'accueil étant mal différenciée, leur inadaptation relève souvent d'une imbrication difficile à démêler et étrangement proche du "double bind " défini par l'école de Palo Alto. Ces cas, auxquels on devrait consacrer des études sérieuses, ne rentrent toutefois pas dans le cadre de notre analyse.

Chez les adolescents nés en banlieue parisienne, rares sont les pathologies exprimées sous une forme traditionnelle. Elles peuvent exister ou s'éveiller à l'occasion d'un retour au pays (Mesmin 2000: 77-78) mais, personnellement, je n'en ai encore jamais rencontré. Les adolescents appartenant à la deuxième génération d'immigrés expriment fréquemment leur malaise par les délits typiques, propres à cette classe d'âge : fugues, rackets, agressions sexuelles, "vols en réunion ", trafic et usage de drogue. Ces actes qui ne visent souvent qu'un succès éphémère, jonglent avec les limites du social proches de la catastrophe, en entretenant un clivage invivable qui, en soi, constitue un signal de détresse. Parler avec les adolescents permet parfois d'approcher leur malaise qui peu à peu révèle une histoire dans laquelle morts par accident, abandons, ruptures brutales, injustices jouent un rôle déterminant. Les représentations de la relation avec les autres sont toujours plus importantes que les expressions proprement culturelles, même si celles-ci méritent de ne pas être négligées. En tout cas, cette approche ne m’a pas amené à retrouver, parmi les enfants d'immigrés, " un noyau onirique " témoignant de la continuité d' " une personnalité ethnique" à travers les générations (Devereux 1996 : 382). Un cauchemar n'est ni soninké ni bété ou baoulé, mais d'abord et avant tout l'expression d'une angoisse propre à un sujet.

Rien ne vient confirmer non plus que «le fondement de la culture repose essentiellement sur l'irrationnel " (ibid. : 306) ou sur «le noyau psychotique que chacun a en soi " (ibid. : 803). Au contraire, j'ai fini par constater que les adolescents traduisaient leurs tourments et leurs revendications dans des formes de culture nouvelles telles que le rap, le hip-hop ou la break-dance. Nombreux sont ceux qui possédent des cahiers de chansons créées par eux afin d'exprimer, sans avoir à l'avouer, une partie de leur " personnalité cachée». Cette culture de pas- 
sage qui fait l'unanimité chez eux a valeur d'une identité et d'une altérité proclamées, l'une n'allant pas sans l'autre. Tel est aussi le cas de l'intégrisme religieux auquel souscrivent quelques-uns. Le port du tchador est à la fois le signe d'un islam affirmé et la marque d'une altérité qu'adopte un sujet pour se distinguer des autres. Ces formes de culture qui se cherchent en bordure du social sont, comme toute expression artistique ou religieuse extrême, une tentative d'amorcer de nouvelles formes de clivage vivables. Elles prouvent que les enfants de la première génération d'immigrés en passe de devenir adultes ne peuvent se construire ni dans la seule culture de leur parents ni dans celle enseignée dans le pays d'accueil, mais dans des formes nouvelles, qui, leur étant propres (appartenance et inclusion), leur permettent de se situer dans leur différence. Comme l'exprime notre exergue, "la situation migratoire constitue en elle-même, dans bien des domaines, une situation de rupture ", un clivage entre un passé et un présent, facteur de changement.

Il existe donc entre les première et deuxième générations d'immigrés africains un décalage d'autant plus fort que leurs clivages respectifs sont inverses. Si les pères appartiennent profondément à leur société d'origine et ne sont qu'inclus dans leur société d'arrivée, leurs enfants, eux, appartiennent surtout à leur société d'accueil et ne sont qu'inclus dans leur société d'origine. Cette inversion, source permanente de conflit générationnel dans les familles d'immigrés, prouve comme Franz Boas l'avait entrevu, que la culture des parents ne se transmet que très partiellement aux enfants. Nous avons aussi relevé que, dans les deux générations, les femmes et les filles, qui savent bénéficier des aides et des formations auxquelles elles ont droit, sont plus intégrées que les hommes dans la société d'accueil. Cette analyse révèle ainsi que les immigrés, représentant par excellence le paradigme de l'autre, sont clivés de différentes manières selon les générations et les genres, et que, dans tous les cas, ces clivages servent plus de normes que les identités affichées, être Africain ou Européen, Malien ou Français. C'est pourquoi les principaux dérapages que nous avons relevés ont été présentés comme résultant d'une absence de clivage et non comme une absence d'identité. Être clivé, ce n'est pas être irrémédiablement divisé mais être suffisamment à distance des modes de vie des sociétés d'origine et d'accueil, pour pouvoir jeter des passerelles de l'une à l'autre. Sans clivage, pas de traduction possible, pas de translittération non plus entre les langues et les conventions sociales.

MOTS CLÉS/KEYWORDS: immigration/immigration - générations/generations - appartenance/ membership - inclusion/inclusion - clivage/cleavage. 
Allouch Jean

1994 Marguerite ou l'Aimée de Lacan.

Postface de Didier Anzieu. Paris, EPEL (1 $1^{\text {re }}$ éd. 1990).

\section{Badiou Alain}

1998 L'être et l'événement. Paris, Éditions du Seuil ("L'Ordre philosophique»).

\section{Bastide Roger}

1970 Le prochain et le lointain. Paris, Cujas.

\section{Collomb, Henri \& Jacques Zwingelstein}

1963 "Psychiatrie en Afrique", ms. multigr., 74 p.

\section{Devereux, Georges}

1982 Psychothérapie d'un Indien des Plaines. Paris, Éditions Jean-Cyrille Godefroy ( $1^{\text {re }}$ éd. 1951).

1996 Ethnopsychiatrie des Indiens Mohaves. Paris, Synthélabo ( penser en rond») (1 ${ }^{\text {re }}$ éd. 1961).

Durkheim, Émile

1913 "Compte rendu de Franz Boas, The Mind of the Primitive Man", L'Année sociologique 12 (1909-1912).

\section{Hansen, Marcus Lee}

1940 The Immigrant in American History. Cambridge, Harvard University Press.

Mesmin, Claude

2000 «La parole qui guérit. Des mots pour chasser les djinns", in Guérir l'âme et le corps. Au-delà des médecines habituelles. Paris, Albin Michel : 69-82.
Ortigues, Marie-Cécile \& Ortigues, Edmond

1976 Edipe africain. Paris, Plon (1 ${ }^{\text {re }}$ éd. 1973).

\section{Quiminal, Catherine}

1991 Gens d'ici, gens d'ailleurs. Paris, Christian Bourgois.

1995 «Les associations de femmes africaines en France. Nouvelles formes de solidarité et individualisation ", Cahier $d u$ Gedisst 21 : 111-130.

1997 "Présentation» in Migrations. Synthèses de travaux sur l'immigration et la présence étrangère en France. Paris, Ministère de l'emploi et de la solidarité : 1-6.

\section{Sayad, Abdelmalek}

1977 «Les trois âges de l'émigration algérienne en France ", Actes de la Recherche en Sciences sociales $15: 59-82$.

\section{Schnapper, Dominique}

1998 La relation à l'autre. Au coeur de la pensée sociologique. Paris, Gallimard («Essais»).

\section{Timera, Mahamet}

1996 Les Soninké en France. D'une histoire à l'autre. Paris, Karthala.

Winthol de Wenden, C.

1988 Les immigrés et la politique. Paris, Presse de la Fondation national des sciences politiques.

Zempléni, András \& Rabain, Jacqueline 1965 «L'enfant nit ku bon, un tableau psychopathologique traditionnel chez les Wolof et Lébou du Sénégal ", Psychopathologie africaine III (3) : 143-177. 